\title{
An Energy-Efficient Adaptive Sensing Framework for Gait Monitoring Using Smart Insole
}

\author{
Yingxiao Wu, Member, IEEE, Wenyao Xu, Member, IEEE, Jason J. Liu, Member, IEEE, \\ Ming-Chun Huang, Member, IEEE, Shuang Luan, Member, IEEE, \\ and Yuju Lee, Student Member, IEEE
}

\begin{abstract}
Gait analysis is an important process to gauge human motion. Recently, longitudinal gait analysis received much attention from the medical and healthcare domains. The challenge in studies over extended time periods is the battery life. Due to the continuous sensing and computing, wearable gait devices cannot fulfill a full-day work schedule. In this paper, we present an energy-efficient adaptive sensing framework to address this problem. Through presampling for content understanding, a selective sensing and sparsity-based signal reconstruction method is proposed. In particular, we develop and implement the new sensing scheme in a smart insole system to reduce the number of samples, while still preserving the information integrity of gait parameters. Experimental results show the effectiveness of our method in data point reduction. Our proposed method improves the battery life to $10.47 \mathrm{~h}$, while normalized mean square error is within $10 \%$.
\end{abstract}

Index Terms-Gait analysis, pressure sensors, smart insole, sparse representation, selective sampling, image reconstruction.

\section{INTRODUCTION AND BACKGROUND}

G AIT is one of the important characteristics of human locomotion, and gait analysis has been applied in diverse applications in the medical and healthcare domains [1]. Specifically, in elderly healthcare, gait analysis is an important tool to assess fall risk and fall prevention [2], [3]. As an example, Baker used Brand's four reasons to confirm that gait analysis is a useful method for clinical rehabilitation [4], [5]. In addition, gait parameters of post-stroke patients were analyzed to evaluate their rehabilitation status [6]. In the fields of orthopedics and prosthetics, gait analysis is essential to quantify gait conditions and assess the applications of orthopedic assistive devices [7].

Gait labs are the traditional environments where medical practitioners observe human gait motions. These controlled

Manuscript received November 10, 2014; revised November 12, 2014; accepted November 12, 2014. Date of publication November 20, 2014; date of current version February 10, 2015. This work was supported by the U.S National Science Foundation under Grant CNS-1423061. The associate editor coordinating the review of this paper and approving it for publication was Prof. Subhas C. Mukhopadhyay.

$\mathrm{Y}$. $\mathrm{Wu}$ and $\mathrm{W}$. $\mathrm{Xu}$ are with the Department of Computer Science and Engineering, University at Buffalo, State University of New York, New York, NY 14260 USA (e-mail: wenyaoxu@buffalo.edu).

J. J. Liu is with the Department of Computer Science, University of California at Los Angeles, Los Angeles, CA 90095 USA.

M.-C. Huang and Y. Lee are with the Department of Electrical Engineering and Computer Science, Case Western Reserve University, Cleveland, OH 44106 USA.

S. Luan is with the Department of Computer Science, University of New Mexico, Albuquerque, NM 87131 USA.

Color versions of one or more of the figures in this paper are available online at http://ieeexplore.ieee.org.

Digital Object Identifier 10.1109/JSEN.2014.2372694 systems are equipped with high-speed cameras, pressure sensitive carpets and lighting facilities. In a gait study, the subject will walk with reflective markers on the body, and these devices will capture the motion and allow extraction of the subject's gait parameters. With the development of wearable sensor systems, it makes sense to move the monitoring outside the lab, where longitudinal gait monitoring can be done in a more comfortable and realistic setting. Mobiles systems with 3-axis accelerometers and gyroscopes are suitable devices for data collection and analysis [8]; Bae et al. [9] presented a Force Sensing Resistive (FSR) sensor array based system for gait analysis; and Chen et al. [10] used inertial sensors on cerebral palsy patients for longitudinal motion assessment. Martínez-Martí et al. [11] uses a pair of instrumented insoles which can measure pressure from different points of the foot. The insoles have four commercial piezoresistive pressure sensors and a three-axis accelerometer to determine foot forces during stance and swing phases. Flexible conductive textiles can be integrated with many kinds of wearable devices to collect detailed information on complex user activities. Through adjusting E-field frequency and positions of sensors, analyzable signal range and sensitivity of conductive textiles can be improved [12]. A physiological parameters monitoring system with Zigbee based devices can help patients live on their own and minimize the danger of falls or stroke. When emergency events happen, other people can receive notifications to provide help immediately [13]. Measuring human joint movement with fiber-optic curvature sensors can be used for the medical and sport application. These sensors can be put on wearable devices and are noninvasive and safe [14].

In this paper, we present our recent work on designing a gait monitoring system, called Smart Insole. In extending prior work [15], this platform consists of a $52 \times 20$ pressure sensor array. The system can offer high spatial-resolution sensing of plantar pressure from which gait features can be extracted. However, power consumption is the main challenge because of the concentration of sensors. In general, there are two options: either reduce the sampling rate or reduce the sampling density. To this end, we propose a new adaptive sensing scheme to reduce the sampling density, as well as control the locality of samples, while preserving information fidelity. To gauge the sparsity of spatial sensor data, we effectively reduce the number of sample points needed in the system. Compared to the previous compressed sensing technology [16], our method can be implemented with the traditional ADC modeling rather 


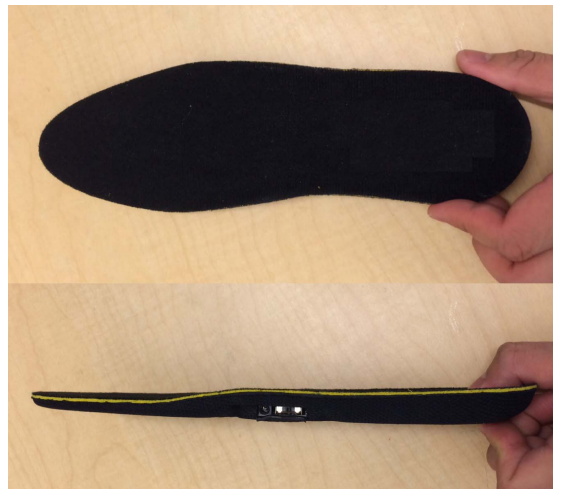

(a)

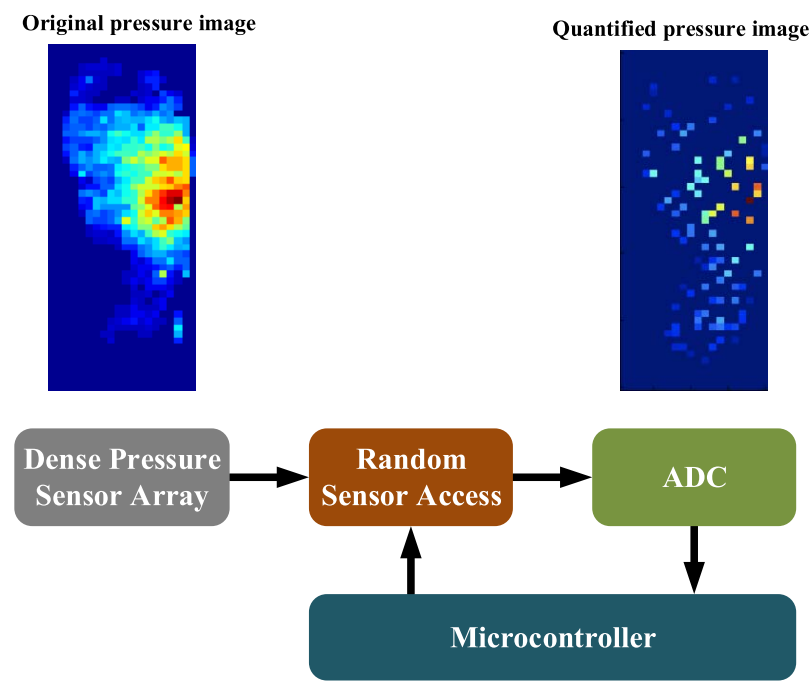

(b)

Fig. 1. Smart Insole system for gait monitoring. There is a $52 \times 20$ pressure sensor array in the device. The embedded microcontroler randomly samples from the dense pressure sensor array and only these pixels what have been processed by ADC are used for reconstruction. (a) Smart Insole. (b) System Structure.

than requiring special ADC components. In the reconstruction of the high density image, we claim that our proposed method of sparse representation is better than simple interpolation especially for low number of samples, as illustrated in our experimental results.

The remainder of the paper is structured as follows. Section II describes the design and implementation of the Smart Insole system. Then we present the adaptive sensing framework which reduces the number of selected samples and reconstructs data in Section III. In Section IV, we discuss the experimental results. We conclude in Section $\mathrm{V}$ and outline the future research direction and related clinical applications.

\section{SMART InSOLE}

\section{A. System Description}

In this section, we introduce the Smart Insole system we developed. As illustrated in Fig. 1(a), Smart Insole looks like a normal insole equipped with $52 \times 20$ pressure sensors. Fig. 1(b) illustrates the schematic structure of the Smart
Insole system. A microcontroller controls a random sensor access unit that reads the target sensor. The sensor signal is quantified by an ADC module and then saved in a data storage unit, such as an SD card. The entire electronic system is powered by a $400 m A h$ lithium battery. The pressure sensor is implemented with e-Textile which is a fiber-based yarn coated with piezoelectric polymer, e-Textile has large process variation and can be designed as a high-density and low-cost pressure sensor array. The dense pressure sensor array homogeneously covers the surface of insole and is implemented with flexible print-circuit board. The details are given in our related work [17]. Using this device will not cause discomfort which enables longitudinal gait monitoring in everyday life, i.e. in uncontrolled environments.

\section{B. Energy Challenge}

The main challenge in using Smart Insole for longitudinal gait monitoring is energy consumption. Due to the limitation of size and weight, there is only room to fit a $400 \mathrm{mAh}$ lithium battery. To ensure the fidelity of gait information, the sample rate of the pressure sensor array in gait analysis is $30 \mathrm{~Hz}$. A prior study shows that the system can last for 2 hours, which is not sufficient to support a full-day's worth of longitudinal monitoring. The most effective way to save energy for longer battery life is to reduce the number of sample points. In the next section, we will present an energy-efficient adaptive sensing method.

\section{OUR FRAMEWORK}

In this section, we introduce our proposed energy-efficient adaptive sensing framework in Smart Insole. Fig. 2 shows the scheme overview. More specifically, there are three main components in this framework: pre-sampling and real-time gait analysis, selective sensing, and sparsity-based signal reconstruction. The first two components occur in the Smart Insole device, while the third component runs in the host system. The sensed data is recorded locally on an SD card or transmitted to the remote host for reconstruction and gait feature analysis. We will elaborate on these components in the following sub-sections.

The general idea of adaptive sensing is to change the number of samples for each frame according to the pressure image content. For example, there are four different gait stages in one gait cycle: swing (S), contact (C), midstance (M) and propulsive $(\mathrm{P})$ stage [18]. Fig. 3 shows the pressure image in each stage. We can see that the pressure distribution on each stage is different, and it can use selected pressure sensors to determine the gait phase. For instance, there is no pressure under the foot in the swing stage, and there is no need to sample the pressure image.

\section{A. Pre-Sampling and Real-Time Gait Cycle Analysis}

The first part is to analyze the gait stage. There are two considerations here. First, the analysis procedure should run in real-time for the sake of high speed sampling. Second, the analysis should be accurate to ensure the correctness of gait 


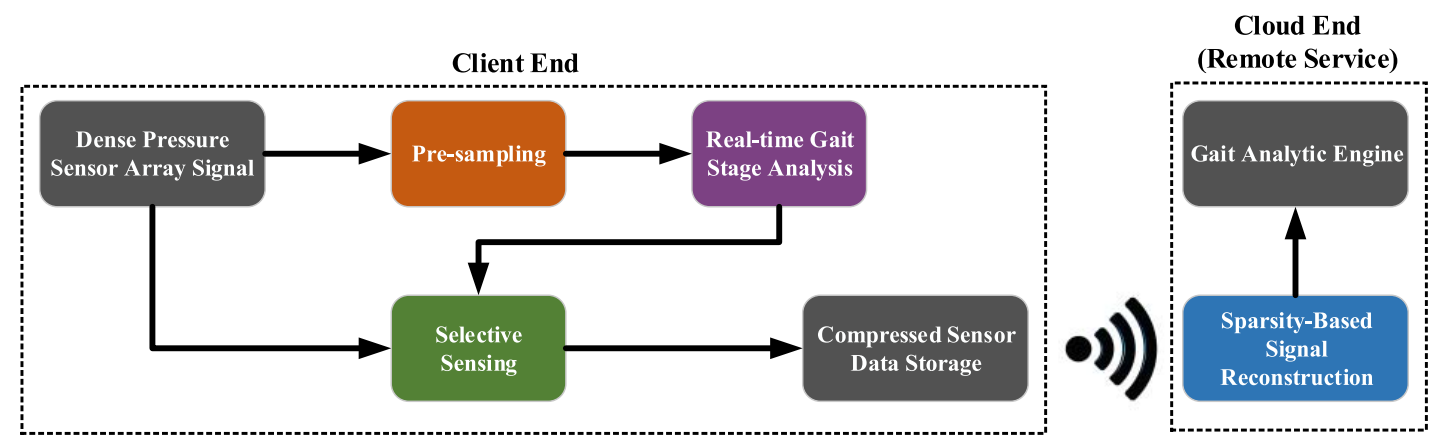

Fig. 2. Overview of energy-efficient adaptive sensing framework in Smart Insole. The components in the client end are pre-sampling and real-time gait analysis, selective sensing, and sparsity-based signal reconstruction. The sensing data can be transmitted to the cloud end for reconstruction and gait feature analysis.

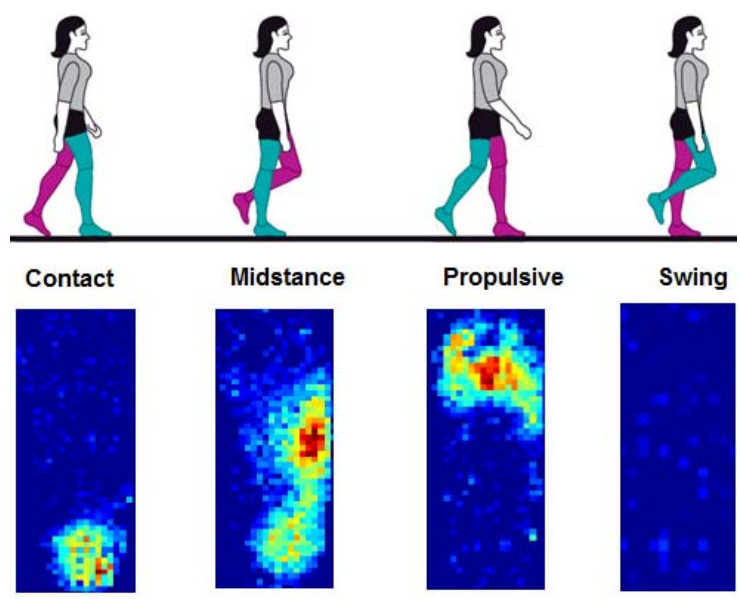

Fig. 3. Four stages in one gait cycle: contact, midstance, propulsive and swing. The corresponding pressure images are under each.

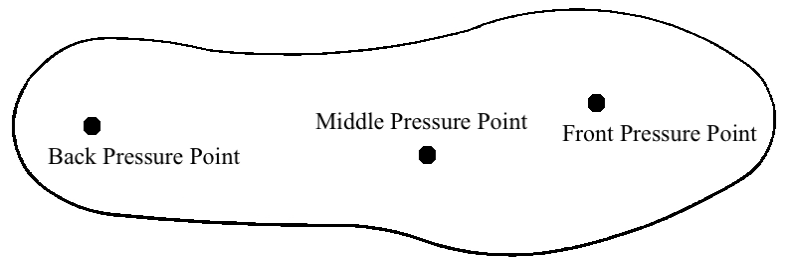

Fig. 4. Three pre-sampling points: Front point (FP), Middle point (MP) and Back point (BP).

cycle analysis. In this work, we pre-sample three prominent points in the pressure sensor array to recognize the gait phases. As shown in Fig. 4, three positions are at the front, middle and back, respectively. In this way, we can use three easily sampled sensor values to gauge the stage phase using the process as described in Algorithm 1. We estimate the gait stage according to the pressure threshold (Th). Specifically, if the pressure of Front point (FP) is larger than threshold, at the same time the pressure of Back point (BP) and Middle point (MP) is less than threshold, then gait stage is Propulsive; if the pressure of FP and MP is less than threshold, at the same time the pressure of BP is larger than threshold, then gait stage is Contact; if the pressure of FP, MP and BP is larger than threshold, then gait stage is Midstance; if the pressure of FP, MP and BP is less than threshold, then gait stage is Swing.

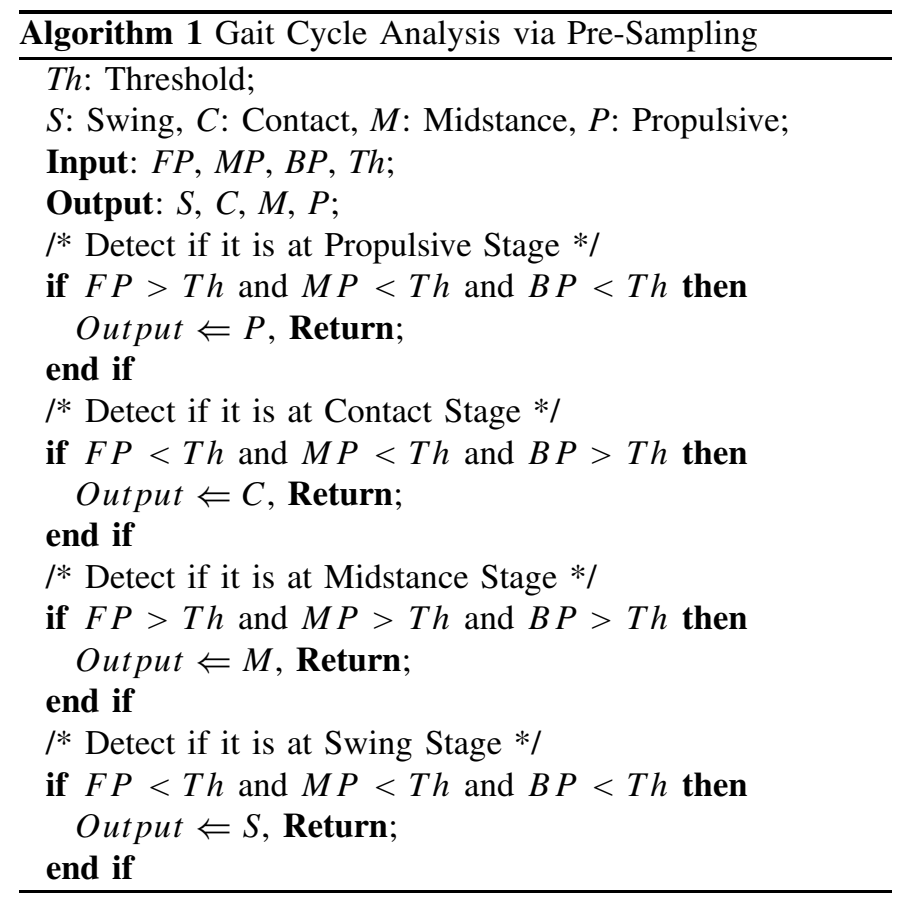

\section{B. Local Randomized Selective Sensing}

The second step is selective sampling for dense pressure image. The goal of selective sampling is to reduce the sample number per frame; however, the system still can estimate and reconstruct the entire pressure image by these limited sensor measurement points. With support from our preliminary study (shown in the experimental part), the structure of pressure image varies in different gait stages. Given that the system identifies which gait stage the subject is in by Algorithm 1 in the previous subsection, we are able to apply a two-fold sampling strategy. Firstly, the samples are selected randomly and sparsely. Secondly, the sparse sampling is limited to regions around predefined locales. The process is described here.

For one pressure image frame, we consider an image $x$ as a $N$ pixel vector in $\mathbb{R}^{N}$, i.e. the two dimension image is unfolded into a single column vector. This image $x$ can be represented as a matrix form:

$$
x=I x,
$$

and where $I$ is the $N \times N$ identity matrix. 
We perform row-wise swaps on the identity matrix to convert $I$ to $A$ so that $A$ is a randomized permuted version of $I$ :

$$
A_{N \times N}=\left[\begin{array}{ccccccc}
0 & \ldots & \ldots & \ldots & 1 & \ldots & 0 \\
0 & \ldots & 1 & \ldots & \ldots & \ldots & 0 \\
\ldots & \ldots & \ldots & \ldots & \ldots & \ldots & \ldots \\
0 & \ldots & \ldots & 1 & \ldots & \ldots & 0
\end{array}\right],
$$

Now $y$ is informatively equivalent to $x$ in

$$
y=A x .
$$

When the top $k$ rows, $A_{k}$, of $A$ are selected to construct $y$, denoted as $y_{k}$, we have:

$$
y_{k}=A_{k \times N} x .
$$

We can see that $y_{k}$ is not informatively equivalent to original $x$ since $N-k$ sensor values are missing. We call $A_{k \times N}$ a selective sensing matrix, and $y_{k}$ is the selective sensing output. This process describes how the random selection of samples is made. The only update that is needed to restrict the sampling to localized regions is to predefine the allowable permutations of rows of $A$, i.e. only certain rows of $A$ are allowed to be swapped. This ensures that the top $\mathrm{k}$ rows will be sampled from the specific regions. Note that the value of $k$ will be set according to the different gait stages. The results will be presented in Section IV.

\section{Sparsity-Based Reconstruction}

In this subsection, we will discuss how to use $y_{k}$ to reconstruct image $x$. Our reconstruction method is based on the theory of sparse representation (SR). Sparse presentation has been successfully applied to signal reconstruction, i.e. in Compressed Sensing [19]. In Compressed Sensing, $x \in \mathbb{R}^{N}$ is a vector of unknown variables, and $y \in \mathbb{R}^{k}$ is the limited measurements through $A \in \mathbb{R}^{k \times N}$. $A$ is typically either a random Gaussian or Bernoulli matrix. Then, we have:

$$
y=A x,
$$

Since $k$ is less $N$, Eq. (5) becomes an underdetermined system, and $x$ can not be uniquely reconstructed. However, if $x$ is a structural signal, it should be sparse under some transformation $\Psi$, i.e. $x=\Psi z$, where $z \in \mathbb{R}^{N}$ is sparse. In this way, we can reconstruct $x$ with the $l_{0}$ minimization formulation:

$$
\begin{aligned}
& \min _{z}\|z\|_{0} \\
& \text { s.t. } y=A \Psi z .
\end{aligned}
$$

With Eq. (6), there are two questions here. The first question is how to find the transformation $\Psi$ such that $z$ is sparse. In our application, considering $x$ is a pressure image measurement, we employ the direct cosine transform (DCT) to represent the unknown $x$.

The second question is how to solve this formulation. Eq. (6) is a determined system and has stable solution. However, Eq. (6) is an intractable $N P$-hard problem [20].

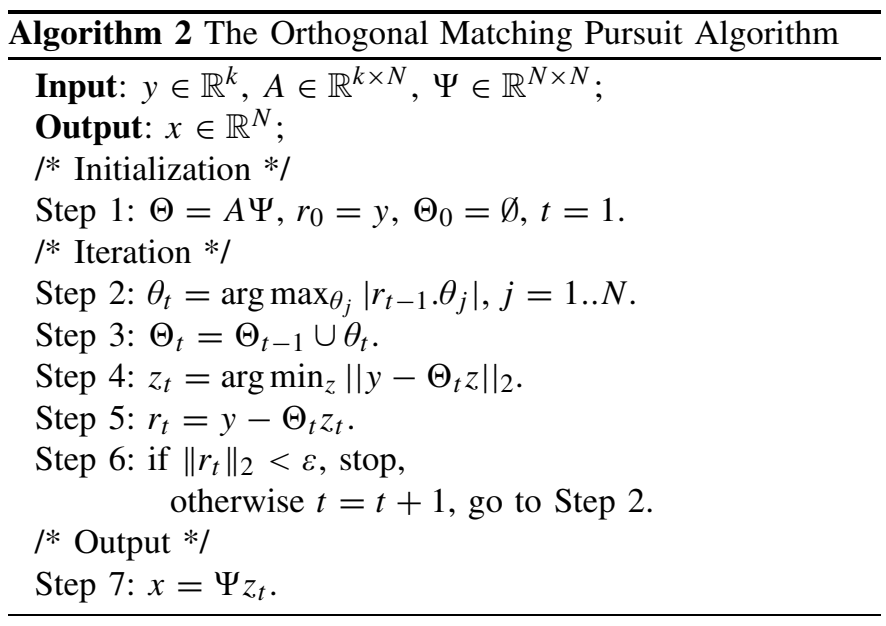

There are two ways to estimate Eq. (6). One is to covexify the formulation with the $l_{1}$ minimization [16]:

$$
\begin{aligned}
& \min _{z}\|z\|_{1} \\
& \text { s.t. } y=A \Psi z .
\end{aligned}
$$

However, solving the $l_{1}$ minimization is time-consuming and not scalable [16]. The other method uses heuristic algorithms to approximate the solution of $l_{0}$ directly. One of the popular methods is Orthogonal Matching Pursuit (OMP) [21]. OMP, being a greedy algorithm, has advantages in speed and ease of implementation [22]. If we consider $A \Psi$ as a matrix $\Theta$, then we want to find the sparsest solution $z$ that combines with the columns of $\Theta$ to give $y$. Another way to view this is that the sparse solution is the same solution that would result from a reduced set of columns, say $\Theta_{t}$. OMP finds these columns, or bases, in order by choosing a column of $\Theta$ that most closely resembles $y$ and subtracts off its contribution from $y$ for the next iteration. The details of OMP are described in Algorithm 2.

For more details, the initialization step sets a residual $r_{0}=y-\Theta_{t} z_{t}=y$ for time $t=0$. At iteration $t$, Step 2 finds the column $\theta_{t}$ that maximizes its inner product with $r_{t-1}$ for $j=1 . . N$. This column is the most similar to the residual $r_{t-1}$. Step 3 adds this column vector $\theta_{t}$ to a matrix of chosen bases $\Theta_{t}$, where $\Theta_{0}$ was initialized as an empty matrix. Step 4 finds a $t$ element solution of the system using only $t$ columns. Since $t$ is less than $k$, the solution can be found by least squares. Step 5 calculates new approximations of the residual. $z_{t}$ is the solution of $z$ with the most representative columns of $\Theta$. The residual is always orthogonal to the chosen bases $\Theta_{t}$ which results in a zero correlation between residual and any of these bases, and so future iterations will never choose a previously selected column. This continues until a stopping criterion is met. Finally, Step 7 gives the reconstructed solution for $x$ where $z_{t}$ is transformed by $\Psi$.

\section{EXPERIMENTS AND DISCUSSION}

In this section, we discuss the evaluation of our proposed method. For the sake of the comprehensive study, we develop the experiments and validate the system performance on these aspects, including (A) Evaluation of signal reconstruction 
with variation in sampling sparsity, (B) Energy efficiency improvement, and (C) Information integrity of gait features. The experimental results and discussion will be elaborated in the following sub-sections.

Firstly, we introduce two metrics to quantitatively measure the reconstruction errors:

- NMSE: Normalized Mean Square Error is given by

$$
\frac{\|x-\hat{x}\|_{2}^{2}}{\|x\|_{2}^{2}}
$$

where $x$ represents the original signal, and $\hat{x}$ is the reconstructed image of $x$. NMSE is between 0 to $100 \%$, and it has been widely applied to error quantization in various signals, such as time series [23], images [24] and videos [25].

- SSIM: Structural SIMilarity (SSIM) is often considered a better visual estimator of spatially structural signal similarity, such as images, than NMSE [24]. The main premise is that structural information of image pixels have strong inter-dependencies especially when they are spatially close. The SSIM metric ranges between $[-1.0,+1.0]$ where +1.0 means tested images are identical. In our work, we use the definition and implementation detail from [24].

\section{A. Evaluation of Signal Reconstruction}

We firstly look at the performance on a single pressure image. Fig. 5(a) is the original pressure image from the midstance stage, and Fig. 5(b) is the reconstructed image from $25 \%$ of sensor values randomly selected. The DCT coefficients of the original and the reconstructed are shown in Fig. 5(c) and Fig. 5(d), respectively. The results indicates that the reconstructed image is visually close to the original. It is because the pressure image is intrinsically very sparse under some transform domain (such as DCT in this case), and it is possible to reconstruct the information with limited measurements.

To validate that the method of image reconstruction using Sparse Representation, we compare it with simple image interpolation. Figure 6 shows the comparison of reconstruction error for these methods over 500 image frames for selected samples $k$ between 5-35\%. Image interpolation calculates the unsampled pixels by taking the average values of horizontal and vertical linear interpolations. For high sparsity (low number of samples), this result shows that the Sparse Representation method can reconstruct the images better than pixel interpolation. Furthermore, localized sampling with Sparse Representation reduces the reconstruction error compared to the global version of this method.

We are interested in the method performance on continuous sensing signal of gait cycle. In this part, we use the data of 5 steps of the right foot, including 500 sequential pressure images. The sample rate of Smart Insole is $30 \mathrm{~Hz}$. Figure 7 shows SSIM results in this experiment. The results consist of three curves, which corresponds to the sensing percentage $(k) 25 \%, 50 \%$ and $75 \%$, respectively. It is not surprising that the high sensing percentage leads to higher

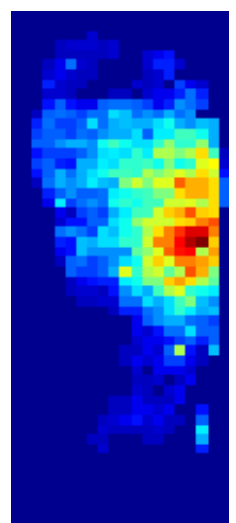

(a)

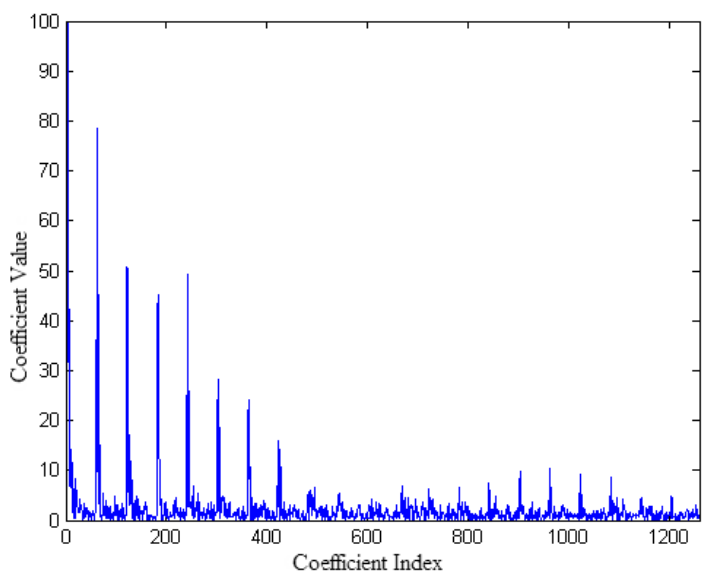

(c)

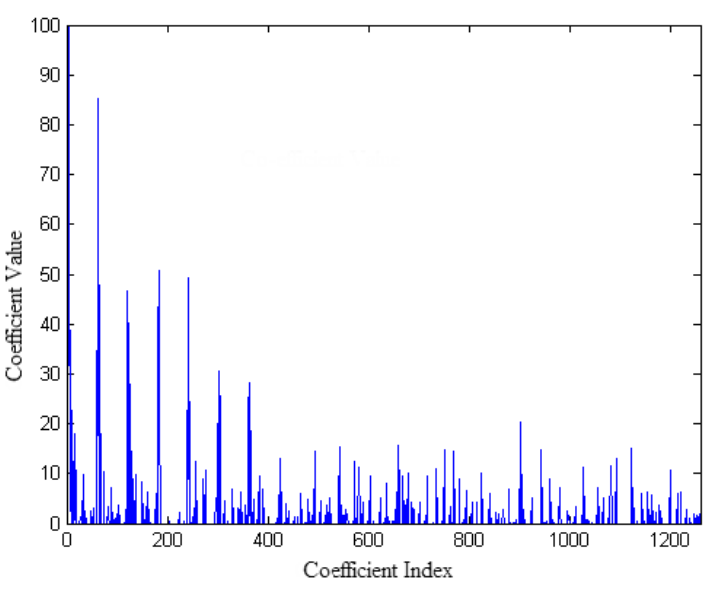

(d)

Fig. 5. Original image versus Reconstructed with $k=25 \%$. Lower graphs show direct cosine transform coefficients. (a) Original. (b) Reconstructed. (c) DCT coefficients original. (d) DCT coefficients reconstructed.

SSIM values. Furthermore, we also see under the constant sensing setup, the reconstruction quality follows some pattern and varies a lot according to different gait stages. More specifically, at the Contact stage, i.e. as the heel touches down, the structural similarity between original and reconstructed images is relatively low (see Fig. 7). It rises through the Midstance to a peak SSIM, then lowers until the Propulsive 


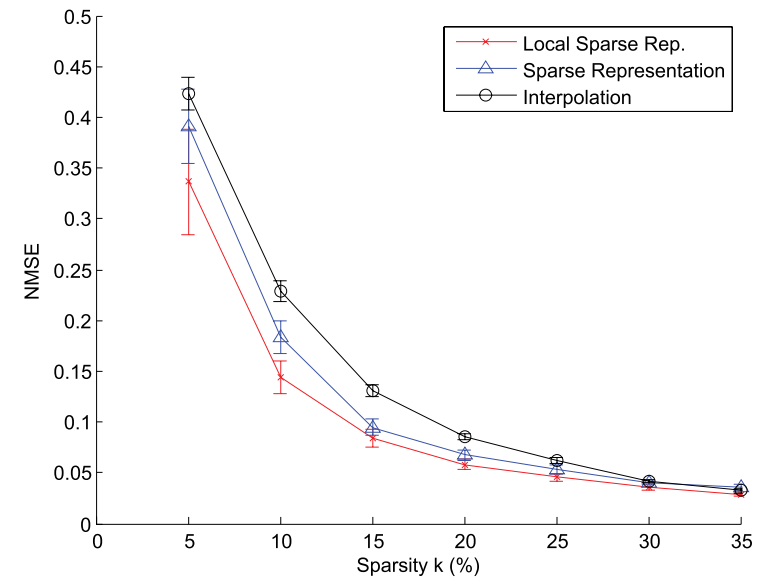

Fig. 6. Reconstruction error for various $k$-selective sensing schemes over 500 sequential reconstructed images for selected sample $K$ between $5 \%$ $35 \%$. Local sparse representation method can reconstruct the images better than pixel interpolation and sparse representation method.

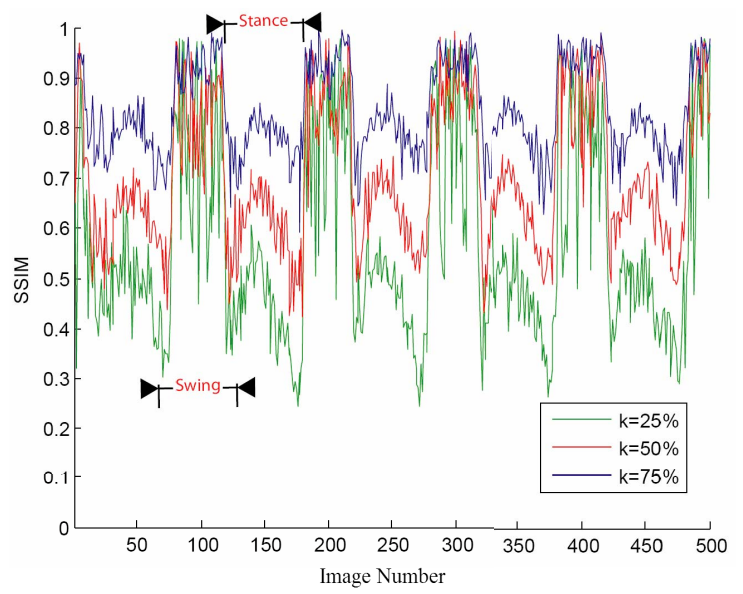

Fig. 7. SSIM for various $k$-selective sensing schemes over 500 sequential reconstructed images.

stage, i.e. as the foot pushes off its ball and toes. In the mean while, we can see that the reconstruction quality in the swing stage is better and more stable than stages in the stance phase. Also, a significant periodicity pattern of SSIM among different sensing setups can be observed in Fig. 7.

Furthermore, we would like to closely look at sample number reduction. We fix the setup of SSIM values and investigate how many samples are required to keep the SSIM values constant. In this experiment, we set up SSIM with four different values, including $0.4,0.5,0.6$ and 0.7 . For the clarity of presentation, we use the data of the stance phase from 10 gait cycles and partition the whole phase into ten segments. The results are shown in Fig. 8, which illustrates how the value of $k$-selective sensing setup varies when structural similarity requirement is constant throughout the step cycle. From the mean value curve, we can see that the higher SSIM, the more required samples. Also, the variation pattern of $k$-selective sensing setup follows the similar trend. More specifically, at the beginning of the step, the value of $k$ is high in order to achieve the required structural similarity to the original image.

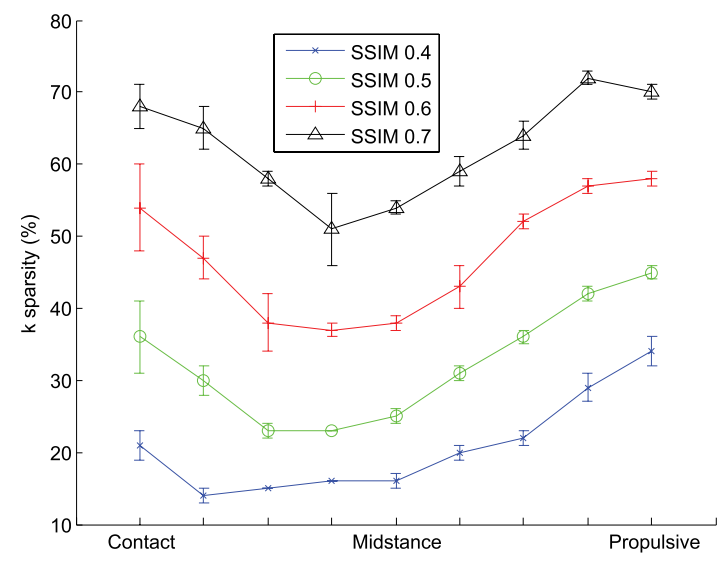

Fig. 8. $\quad k$ selected samples versus phase cycle for various values of SSIM. Error bars indicate range of $k$ values within 0.01 SSIM.

TABLE I

SENSING ENERGy SAVINGS ESTIMATE

\begin{tabular}{|c|c|c|}
\hline NMSE & Sparsity & Battery Life* \\
\hline $0 \%$ & $100.0 \%$ & $2.0 h r$ \\
$1 \%$ & $69.0 \%$ & $2.89 h r$ \\
$2 \%$ & $45.3 \%$ & $4.44 h r$ \\
$5 \%$ & $26.2 \%$ & $7.63 h r$ \\
$10 \%$ & $19.1 \%$ & $10.47 h r$ \\
\hline \multicolumn{2}{|c|}{ * }
\end{tabular}

Toward the middle of the step, the $k$ value can decrease as reconstruction ability improves due to more plantar pressure. Then the $k$ value rises to the end of the step cycle, meaning that more selected samples are required to reach the same level of reconstruction quality. The start and end phases of the step have less structural information in the visual image and so a higher number of selected samples are needed. Note that the standard deviation at the beginning stage is large, and progressively becomes smaller. The observation indicates the large variation of pressure distribution on the heel strike, which can be considered in our future work.

\section{B. Energy Analysis}

The important piece of evaluation is the energy efficiency improvement that this method provides. Table I shows the estimated battery life extension for variations in the reconstruction error. This is based on an initial estimate of $2 \mathrm{hrs}$ battery life of a $400 \mathrm{mAh}$ battery. The energy savings is calculated from the expected sparsity for each reconstructed frame at a certain error value for NMSE.

To further quantify the impact of $k$-selective sensing on different gait stages and its effect on power consumption, we investigate the pressure images from Contact, Midstance and Propulsive stages. Fig. 9 shows the NMSE values of these three images along with different $k$ values ( $k$ is the selected sampling percentage). We can see that the information quality varies a lot with the change of sensing percentage in all three gait stages. Specifically, the image of Midstance always has low reconstruction error compared to the other two stages. It is because the foot pressure in Midstance is more widely distributed and forms a more structural shape. Also, we notice 


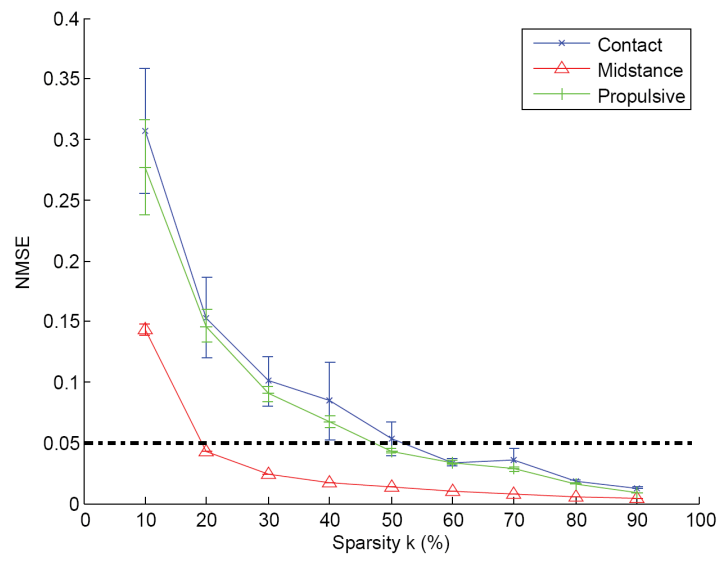

Fig. 9. NMSE versus $k$ selected samples for frames at Contact, Midstance and Propulsive stages. Error bars indicate variance across 500 frames.

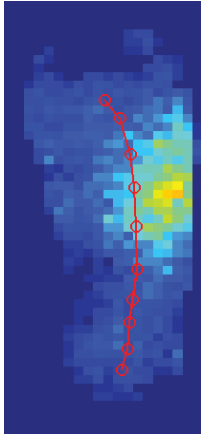

(a)

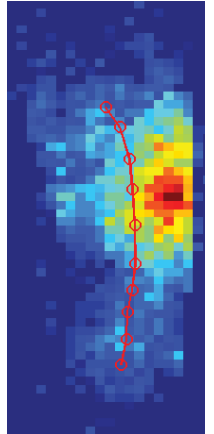

(b)

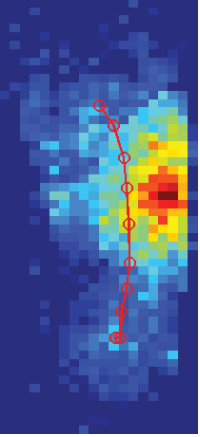

(c)
Fig. 10. Trajectories of calculated center of mass of pressure image sequence with different SSIM values using Local Randomized Selective Sensing. (a) Original SSIM 1.0. (b) Reconstructed SSIM 0.7. (c) Reconstructed SSIM 0.6.

that the performance on Contact and Propulsive is close to each other in the entire $k$-selective sensing test. The result indicates that given a threshold of NMSE, we can reduce a fairly large number of samples according to different gait stages. For example, only $20 \%$ of sensor measurements are needed to reach 0.05 of NMSE in Midstance, but correspondingly 50\% of sensor measurements are required in Contact and Propulsive. Accordingly, this gives evidence that the value of $k$ can be adjusted through the gait cycle to allow a good trade off between reconstruction accuracy and power consumption of sensing.

\section{Information Integrity on Gait Features}

Finally, we would like to investigate the impact of $k$-selective sensing on gait features. In this experiment, we use the pressure image data of one gait cycle with SSIM 1.0, 0.7 and 0.6. For simplicity, we choose one important gait feature, center of mass trajectory also known as gait-line [26], for the evaluation. Figure 10 shows representative results of the center of mass trajectory calculated from the reconstructed images. Specifically, the red circle is the center of mass position in each pressure image, and the red curve is the trajectory formed by center of mass positions in the sequential pressure images. We can see that although the calculated

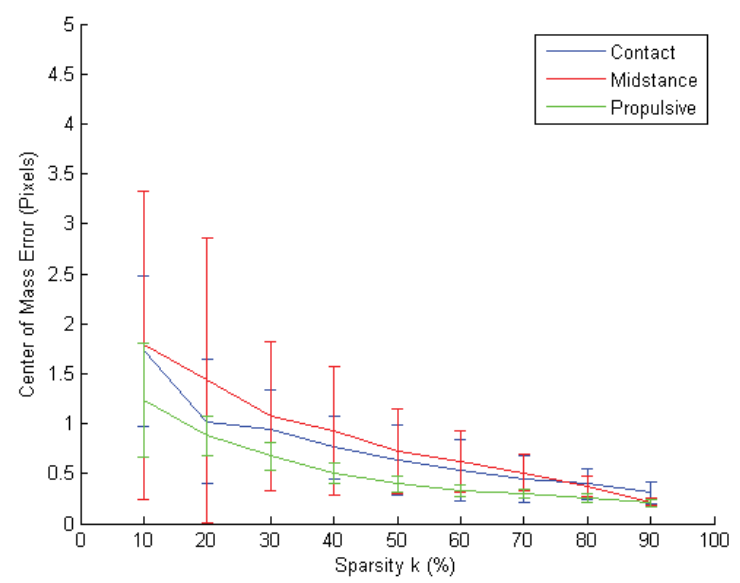

Fig. 11. Measure of Center of Mass (Gait-line) error. This gait feature maintains a low error with variation in sparsity.

TABLE II

Estimated Gait Feature ACCURACy

\begin{tabular}{|c|c|c|}
\hline Feature & Sparsity & Accuracy \\
\hline Gait-line, center & $20 \%$ & $94.3 \%$ \\
of mass & $10 \%$ & $90.7 \%$ \\
\hline Gait-line velocity & $20 \%$ & $93.6 \%$ \\
& $10 \%$ & $91.1 \%$ \\
\hline Cadence, steps & $20 \%$ & $100 \%$ \\
per minute & $10 \%$ & $100 \%$ \\
\hline Heel strike location & $20 \%$ & $100 \%$ \\
& $10 \%$ & $96.1 \%$ \\
\hline
\end{tabular}

trajectories among different $k$-selective sensing setups are slightly different, it can preserve the important information in the feature, such as trajectory direction, transition and curvature. The result indicates that, in the case that algorithm SSIM is not high (such as 0.6), the features from reconstructed images are still good for gait analysis.

Figure 11 gives a quantitative measure on the error between the calculated trajectory and that of the original data. In many instances, the center of mass is within 1.5 pixels throughout all phases of the step and even for very sparse sampling. This result shows that reconstruction through sparse representation sufficiently maintains the integrity of this gait feature.

Table II shows a summary of gait feature integrity. These results show that the extraction of gait features from reconstructed plantar pressure images do not degrade even with changes in the sampling sparsity.

\section{CONCLUSion AND Future Work}

We proposed an energy-efficient adaptive sensing framework in this paper, and implemented the method in Smart Insole for gait analysis. The method is based on gait cycle analysis, and employ a $k$-selective sensing scheme to reduce the samples. The experimental result shows that our method can effectively reduce the number of samples required per frame and still keep good signal quality and gait parameters after reconstruction. In the future, we will perform the longitudinal study and see how our method can extend the battery life in Smart Insole. We will try to improve and evaluate the method. For example, we can 
consider to recognize the gait stages with finer granularity for further energy-saving. We will also consider the evaluation with different application scenarios and human activities. Furthermore, medical conditions affect the normal gait patterns and the current method would need to be reviewed to handle specific motor cases, such as inverted gait sequences. Moreover, besides gait analysis, our proposed sensing scheme can be applied to other healthcare applications [27], [28] for the longitudinal studies.

\section{REFERENCES}

[1] D. Gouwanda and S. Senanayake, "Emerging trends of body-mounted sensors in sports and human gait analysis," in Proc. 4th Kuala Lumpur Int. Conf. Biomed. Eng., vol. 21, 2008, pp. 715-718.

[2] Q. Li, J. A. Stankovic, M. A. Hanson, A. T. Barth, J. Lach, and G. Zhou, "Accurate, fast fall detection using gyroscopes and accelerometerderived posture information," in Proc. 6th Int. Workshop Wearable Implant. Body Sensor Netw. (BSN), 2009, pp. 138-143.

[3] F. Bianchi, S. J. Redmond, M. R. Narayanan, S. Cerutti, and N. H. Lovell, "Barometric pressure and triaxial accelerometry-based falls event detection," IEEE Trans. Neural Syst. Rehabil. Eng., vol. 18, no. 6, pp. 619-627, Dec. 2010.

[4] R. Baker, "Gait analysis methods in rehabilitation," Neoroeng. Rehabil., vol. 3, no. 4, pp. 1-10, Apr. 2006.

[5] M.-C. Huang et al. "Intelligent frozen shoulder rehabilitation using virtual reality," IEEE Intell. Syst., vol. 29, no. 3, pp. 22-28, Jun. 2014.

[6] X. Xu, M. Batalin, Y. Wang, and W. Kaiser, "Gait quality evaluation method for post-stroke patients," in Proc. IEEE Int. Conf. Acoust., Speech Signal Process. (ICASSP), Mar. 2012, pp. 613-616.

[7] M. Lusardi, M. Jorge, and C. Nielsen, Orthotics and Prosthetics in Rehabilitation. St. Louis, MO, USA: Elsevier, 2012.

[8] T. Liu, Y. Inoue, and K. Shibata, "Development of a wearable sensor system for quantitative gait analysis," Measurement, vol. 42, no. 7, pp. 978-988, Feb. 2009.

[9] J. Bae, K. Kong, N. Byl, and M. Tomizuka, "A mobile gait monitoring system for gait analysis," in Proc. IEEE Int. Conf. Rehabil. Robot., Kyoto, Japan, Jun. 2009, pp. 50-57.

[10] S. Chen, C. L. Cunningham, B. C. Bennett, and J. Lach, "Enabling longitudinal assessment of ankle-foot orthosis efficacy for children with cerebral palsy," in Proc. ACM 2nd Conf. Wireless Health, San Diego, CA, USA, Oct. 2012, pp. 1-10.

[11] F. Martínez-Martí, M. S. Martínez-García, S. G. García-Díaz, J. García-Jiménez, A. J. Palma, and M. A. Carvajal, "Embedded sensor insole for wireless measurement of gait parameters," Austral. Phys. Eng. Sci. Med., vol. 37, no. 1, pp. 25-35, 2014.

[12] J. Cheng, O. Amft, G. Bahle, and P. Lukowicz, "Designing sensitive wearable capacitive sensors for activity recognition," IEEE Sensors J., vol. 13, no. 10, pp. 3935-3947, Oct. 2013.

[13] K. Malhi, S. C. Mukhopadhyay, J. Schnepper, M. Haefke, and H. Ewald, "A Zigbee-based wearable physiological parameters monitoring system," IEEE Sensors J., vol. 12, no. 3, pp. 423-430, Mar. 2012.

[14] D. Z. Stupar, J. S. Bajic, L. M. Manojlovic, M. P. Slankamenac, A. V. Joza, and M. B. Zivanov, "Wearable low-cost system for human joint movements monitoring based on fiber-optic curvature sensor," IEEE Sensors J., vol. 12, no. 12, pp. 3424-3431, Dec. 2012.

[15] W. Xu, M.-C. Huang, N. Amini, J. J. Liu, L. He, and M. Sarrafzadeh, "Smart insole: A wearable system for gait analysis," in Proc. 5th Int. Conf. Pervasive Technol. Rel. Assist. Environ., Crete, Greece, Jul. 2012, pp. $1-4$.

[16] E. J. Candes, J. Romberg, and T. Tao, "Robust uncertainty principles: Exact signal reconstruction from highly incomplete frequency information," IEEE Trans. Inf. Theory, vol. 52, no. 2, pp. 489-509, Feb. 2006.

[17] W. Xu, Z. Li, M.-C. Huang, N. Amini, and M. Sarrafzadeh, "eCushion: An etextile device for sitting posture monitoring," in Proc. Int. Conf. Body Sensor Netw. (BSN), May 2011, pp. 194-199.

[18] S. A. Dugan and K. P. Bhat, "Biomechanics and analysis of running gait," Phys. Med. Rehabil. Clin. North Amer, vol. 16, no. 3, pp. 603-621, 2005.

[19] D. Donoho, "Compressed sensing," IEEE Trans. Inf. Theory, vol. 52, no. 4, pp. 1289-1306, Apr. 2006

[20] B. K. Natarajan, "Sparse approximate solutions to linear systems," SIAM J. Comput., vol. 24, no. 2, pp. 227-234, Apr. 1995.
[21] Y. Pati, R. Rezaiifar, and P. S. Krishnaprasad, "Orthogonal matching pursuit: Recursive function approximation with applications to wavelet decomposition," in Proc. Conf. Rec. 27th Asilomar Conf. Signals, Syst. Comput., vol. 1, 1993, pp. 40-44.

[22] J. A. Tropp and A. C. Gilbert, "Signal recovery from random measurements via orthogonal matching pursuit," IEEE Trans. Inf. Theory, vol. 53, no. 12, pp. 4655-4666, Dec. 2007.

[23] W. Wei, Time Series Analysis. New York, NY, USA: Addison-Wesley, 1994.

[24] Z. Wang, A. C. Bovik, H. R. Sheikh, and E. P. Simoncelli, "Image quality assessment: From error visibility to structural similarity," IEEE Trans. Image Process., vol. 13, no. 4, pp. 600-612, Apr. 2004.

[25] S. Zhu and K.-K. Ma, "A new diamond search algorithm for fast blockmatching motion estimation," IEEE Trans. Image Process., vol. 9, no. 2 , pp. $287-290$, Feb. 2000.

[26] C. R. Lee and C. T. Farley, "Determinants of the center of mass trajectory in human walking and running," J. Experim. Biol., vol. 13, no. 1 , pp. 2935-2944, Jan. 1998.

[27] W. Xu, M.-C. Huang, N. Amini, L. He, and M. Sarrafzadeh, "eCushion: A textile pressure sensor array design and calibration for sitting posture analysis," IEEE Sensors J., vol. 13, no. 10, pp. 3926-3934, Oct. 2013.

[28] J. J. Liu et al., "Sleep posture analysis using a dense pressure sensitive bedsheet," Pervasive Mobile Comput. J., vol. 10, no. 2, pp. 34-50, Feb. 2014

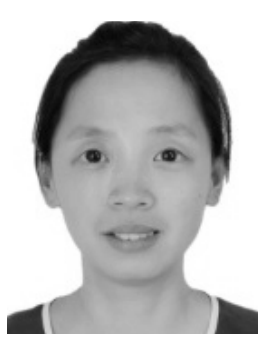

Yingxiao Wu received the $\mathrm{Ph} . \mathrm{D}$. degree from the Department of Communication Engineering, Nanjing University of Posts and Telecommunications, Nanjing, China, in 2010. She is currently a Post-Doctoral Researcher with the Department of Computer Science and Engineering, University at Buffalo, State University of New York, New York, NY, USA. Her current research interests include human-computer interacting, signal processing, and their applications in medical and healthcare.

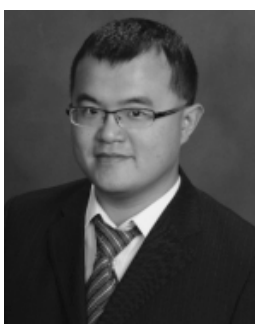

Wenyao Xu (M'08) received the Ph.D. degree from the Department of Electrical Engineering, University of California at Los Angeles, Los Angeles, CA, USA, in 2013. He is currently an Assistant Professor with the Department of Computer Science and Engineering, University at Buffalo, State University of New York, New York, NY, USA. His current research foci include embedded system design and optimization, computer architecture, body sensor networks, mobile health, human-computer interaction, integrated circuit design technologies, and their applications in biomedicine, healthcare, and security. He holds five U.S. and international patents, and has authored over 60 peer-reviewed journal and conference papers.

Dr. Xu was a recipient of the Best Paper Award at the IEEE Conference on Implantable and Wearable Body Sensor Networks in 2013, and the Best Demonstration Award at the Association for Computing Machinery (ACM) Wireless Health Conference in 2011. He is a member of ACM. 


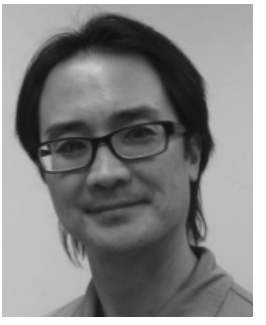

Jason J. Liu received the Ph.D. degree in computer science from the University of California at Los Angeles, Los Angeles, CA, USA, in 2014, the M.S.E. degree in robotics from the University of Pennsylvania, Philadelphia, PA, USA, and the B.E. (Hons.) degree in electrical engineering from the University of Sydney, Sydney, NSW, Australia. He has worked extensively in the industry, in particular, Siemens both in Philadelphia and Sydney.

His research interests include computer vision, machine learning, medical informatics, wireless health, and human-computer interaction. He was a recipient of the Best Paper Award at the IEEE Conference on Implantable and Wearable Body Sensor Networks in 2013.

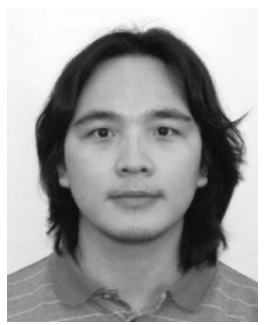

Ming-Chun Huang is currently an Assistant Professor with the Department of Electrical Engineering and Computer Science, Case Western Reserve University, Cleveland, OH, USA. His research interests include wearable computing, big data, Internet of Things, and virtual and augmented reality research. He received the $\mathrm{Ph} . \mathrm{D}$. degree in computer science from the University of California at Los Angeles, Los Angeles, CA, USA. He was a recipient of the Best Paper Award at the IEEE Conference on Implantable and Wearable Body Sensor Networks in 2013 and the Best Demonstration Award at the Association for Computing Machinery Wireless Health Conference in 2011.

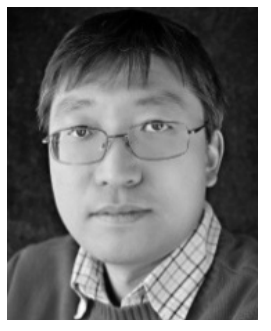

Shuang Luan received the Ph.D. degree from the Department of Computer Science and Engineering, University of Notre Dame, Notre Dame, IN, USA, in 2004. He is currently an Associate Professor of Computer Science and a Joint Associate Professor of Radiology with the University of New Mexico, Albuquerque, NM, USA. His current research foci include biomedical engineering, biomedical informatics, computational geometry, hybrid optimization, and medical embedded systems. He holds five patents and six commercial licensing agreements, and has authored or co-authored over 70 peer-reviewed journal and conference papers.

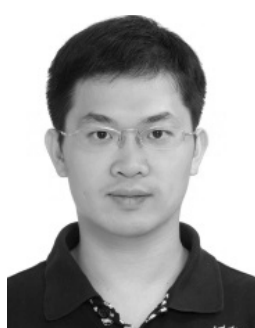

Yuju Lee received the B.S. degree from National Chung Cheng University, Chaiyi, Taiwan, in 2005, and the M.S. degree in electrical engineering from the University of Southern California, Los Angeles, CA, USA, in 2010. He is currently an Engineer with MediaTek, Inc., Hsinchu, Taiwan. His research interests include sensor network systems for medical care, Internet of Things, multiuser MIMO in Wi-Fi, and multiradios coexistence system. 